Case Report

\title{
Pancreaticopericardial Fistula: A Case Report and Literature Review
}

\author{
Muhammad S. Khan, ${ }^{1}$ Najmi Shahbaz, ${ }^{2}$ Hassaan A. Zia, ${ }^{3}$ Muhammad Hamza, \\ Henna Iqbal, ${ }^{2}$ and Ahmed Awab ${ }^{1}$ \\ ${ }^{1}$ Section of Pulmonary \& Critical Care Medicine, University of Oklahoma Health Sciences Center, No. WP1310, \\ 920 Stanton L Young Boulevard, Oklahoma City, OK 73117, USA \\ ${ }^{2}$ Dow University of Health Sciences, Mission Road, Karachi 74200, Pakistan \\ ${ }^{3}$ Department of Internal Medicine, University of Oklahoma Health Sciences Center, No. WP1310, 920 Stanton L Young Boulevard, \\ Oklahoma City, OK 73117, USA
}

Correspondence should be addressed to Muhammad S. Khan; muhammad-khan@ouhsc.edu

Received 17 February 2016; Accepted 20 March 2016

Academic Editor: Kurt Lenz

Copyright (C) 2016 Muhammad S. Khan et al. This is an open access article distributed under the Creative Commons Attribution License, which permits unrestricted use, distribution, and reproduction in any medium, provided the original work is properly cited.

\begin{abstract}
Purpose. Pancreaticopericardial fistula (PPF) is an extremely rare complication of acute or chronic pancreatitis. This paper presents a rare case of PPF and provides systematic review of existing cases from 1970 to 2014. Methods. A PubMed search using key words was performed for all the cases of PPF from January 1970 to December 2014. Fourteen cases were included in the study. The cases were reviewed for demographic characteristics, diagnostic modalities, and treatment. Descriptive analysis of these variables was performed. Results. Median age was 43 years. 78\% were known alcoholics and 73.3\% had chronic pancreatitis. Dyspnea was present in $78 \%$. Cardiac tamponade was present in $53 \%$; $75 \%$ of patients had known chronic pancreatitis $(\mathrm{RR}=0.74)$. Surgery was associated with best treatment outcomes and $50 \%$ of patients who underwent endoscopic treatment survived. Conclusion. PPF is a rare disease. This paper indicates that acute cardiac tamponade in patients with history of alcoholism and chronic pancreatitis could be a sign of an existing pancreaticopericardial fistula and early surgical intervention could be life-saving.
\end{abstract}

\section{Introduction}

Pancreatic pseudocyst is a known complication of pancreatitis. Persistent leakage of pancreatic secretions can result in development of internal fistula due to spontaneous erosion into neighboring hollow viscus and cavities. Leakage from pancreatic secretions can cause significant morbidity due to malnutrition and infections. A fistula from pancreatic pseudocyst to pericardium is a rare complication and can present with cardiac tamponade. In this paper we describe a case of pancreaticopericardial fistula and review similar cases from 1970 to 2014.

\section{Case}

A middle-aged African American male presented to hospital with complaints of shortness of breath and feeling of dizziness for 3 days. Patient had known history of alcoholism and was admitted at a rehabilitation center 3 weeks before presentation for an elective detoxification. His initial course at rehabilitation center was uneventful; however, 3 days before presentation he started feeling dizzy and had gradual onset dyspnea on exertion that progressed to resting dyspnea. This was associated with sharp 3/10 epigastric pain radiating to his chest and back, which was aggravated by deep breaths. His past medical history was also significant for hypertension and episodes of delirium tremens. On admission his temperature was 37 degrees Celsius and blood pressure (BP) was $96 / 48 \mathrm{mmHg}$ after 2-liter fluid bolus in emergency department, with pulse of $110 / \mathrm{min}$, respiratory rate of $20 / \mathrm{min}$, and oxygen saturation of $96 \%$ on $2 \mathrm{~L}$ nasal cannula. Examination was significant for decreased breath sounds in lung bases, increased vocal fremitus in left lower lobe, and dullness to percussion at lung bases bilaterally. 


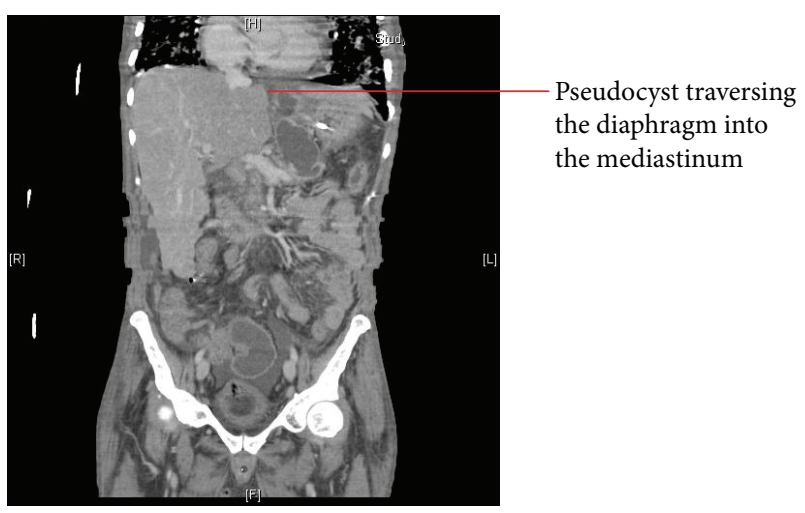

Figure 1: Abdominal CT showing cyst extension into mediastinum.

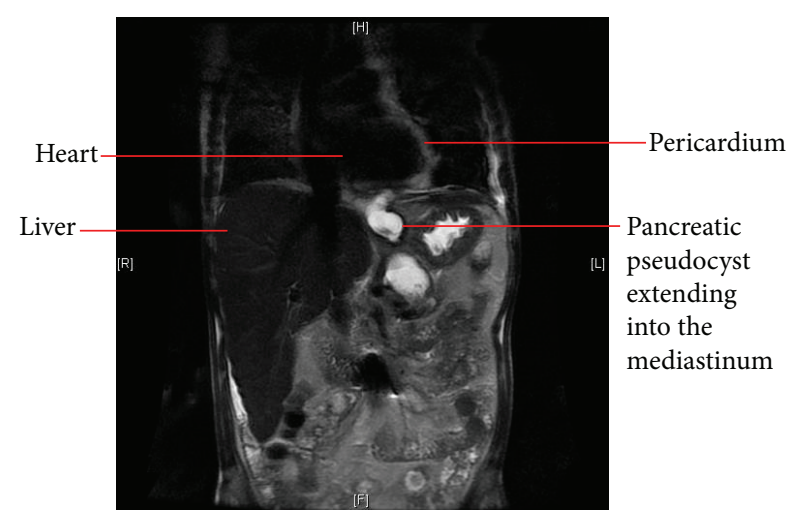

FIGURE 2: MRCP showing cyst extension into the mediastinum.
Jugular venous distension of $7 \mathrm{~cm}$ when sitting and pulsus paradoxus of $15 \mathrm{mmHg}$ were also noted. His white blood cell (WBC) count was $18.5 \mathrm{~K} / \mathrm{mm}^{3}$ (normal range $4-11 \mathrm{~K} / \mathrm{cc}^{3}$ ) and hemoglobin $(\mathrm{Hb})$ was $10.5 \mathrm{~g} / \mathrm{dL}$ (normal range $\mathrm{Hb}$ 13-18 g/dL), with normal electrolytes, liver function tests, cardiac enzymes, and amylase. Due to concern for cardiac tamponade, urgent transthoracic echocardiography was performed that demonstrated a large pericardial effusion with early tamponade physiology. Computed tomography (CT) scan of chest done on admission showed large pericardial effusion and reticular ground glass opacities within the lungs bilaterally. The patient was started on broad spectrum antibiotics and underwent urgent pericardiocentesis and pericardial drain placement, with $280 \mathrm{~mL}$ of serosanguineous fluid obtained. The fluid analysis showed 59500 red blood cells, 3750 white blood cells, lipase of 196 IU, and amylase of $101 \mathrm{IU}$. Pericardial fluid cultures and blood cultures remained negative. On day 2 of admission the pericardial drainage decreased, and the drain was removed. However, after removal the patient acutely became short of breath with oxygen saturation in low 80 s despite a $15 \mathrm{~L}$ nonrebreather mask. He was moved to the medical intensive care unit and intubated. Repeat transthoracic echocardiography showed reaccumulation of pericardial effusion. Due to concern for pulmonary embolism, a CT angiogram of the chest was done, which was negative for pulmonary embolism but showed an abdominal pseudocyst extending from the esophageal hiatus into the mediastinum. This was subsequently followed by a CT of the abdomen, showing necrotizing pancreatitis with multiloculated pseudocyst formation. The largest fluid collection was $4.1 \mathrm{~cm} \times 3.6 \mathrm{~cm}$ traversing the mediastinal plane concerning for pancreaticopleural-pericardial fistula (Figure 1).

To further elaborate the pancreatic cyst and fistula, magnetic resonance cholangiopancreatography (MRCP) was also done which confirmed the findings of a large pseudocyst extending into the mediastinum (Figure 2).

The patient was subsequently extubated and moved to the medicine floor. Endoscopic retrograde cholangiopancreatography (ERCP) was performed to visualize the fistulous tract but was limited due to presence of a calculus in ventral pancreatic duct. Endoscopic ultrasound for cystgastrostomy was attempted and showed a fistulous tract towards the left pleural space. A $4 \mathrm{~cm} 7$ French double-pigtail Zimmon stent was successfully placed into the pseudocyst cavity for decompression. The patient's symptoms improved after this procedure and he was discharged from the hospital on day 14. Unfortunately the patient was lost to follow-up in our clinic.

\section{Materials and Methods}

An extensive PubMed search was performed for all the cases of pancreaticopericardial fistula (PPF) using the key words "pancreatic fistula", "pericardial tamponade", and "chronic pancreatitis" from January 1970 to December 2014. A total of 15 cases were found. Fourteen cases were included in the study; one case was excluded as the pericardial effusion was not from fistulous tract. The cases were reviewed for age; sex; alcohol abuse; past history of chronic pancreatitis; acute pancreatitis on admission; presenting symptoms; associated symptoms of fever, abdominal pain, and chest pain; and hypotension, serum amylase levels, tamponade physiology on echocardiography, pericardiocentesis performed, pericardial amylase levels, diagnostic modality used for confirming the fistula, type of treatment offered, and patient outcomes of recovery versus death. Missing variables were marked as "unknown." Finally a descriptive analysis of the variables was done.

\section{Results}

A total of 15 cases of PPF were found from 1971 to 2014 including our case (Table 1). All reported cases were of male gender. Median age was found to be 43 years. Seventy-eight percent of patients had history of alcoholism; $73.3 \%$ cases had a known diagnosis of chronic pancreatitis. Dyspnea was the chief presenting symptom (78\%) followed by hypotension $(53 \%)$, chest pain (50\%), fever (43\%), abdominal pain (26.6\%), syncope $(6.6 \%)$, weight loss $(6.6 \%)$, and ascites (6.6\%). Acute pancreatitis was present in $42 \%$ of cases. Cardiac tamponade was present in a total of eight cases, was negative in five cases, and was unknown in two cases. Of the patients presenting with cardiac tamponade, $75 \%$ had a past medical history of chronic pancreatitis (relative risk $=0.74$, 


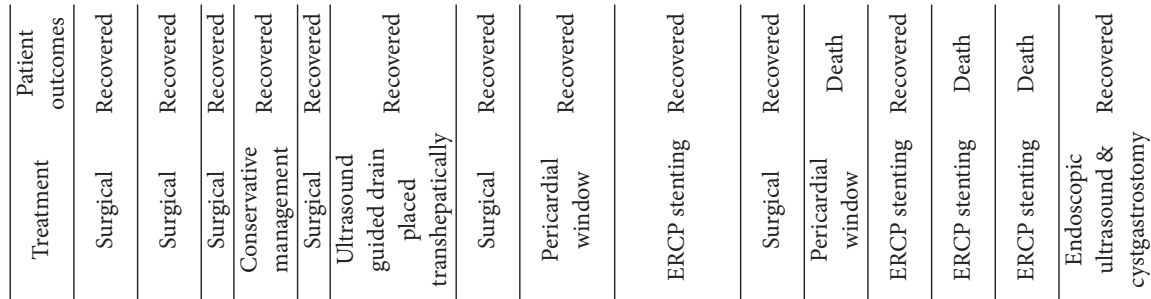

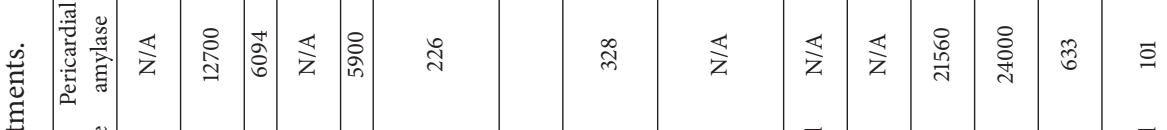

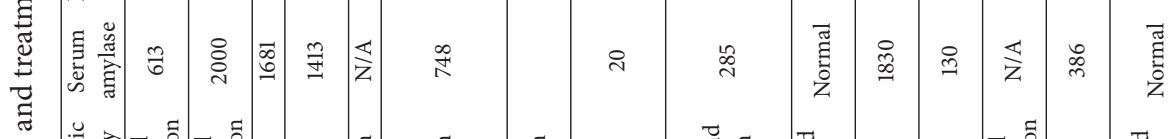

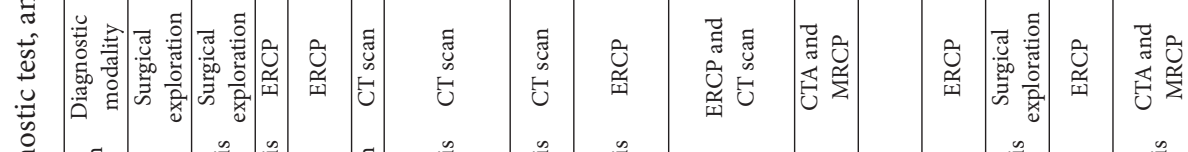

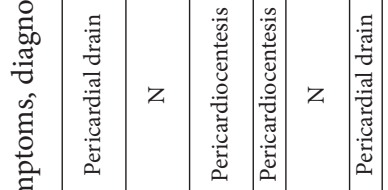

|

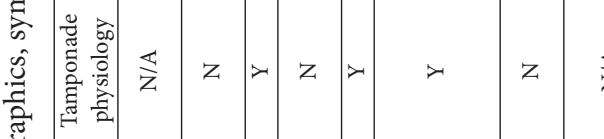

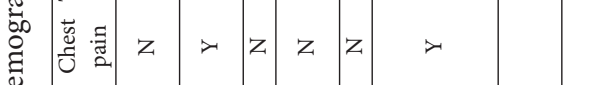

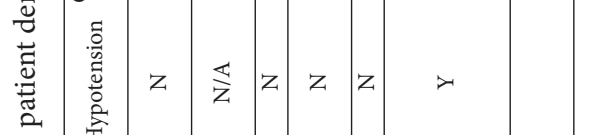

章

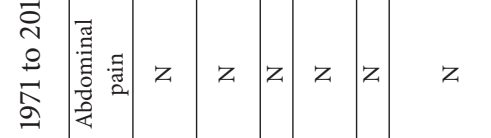

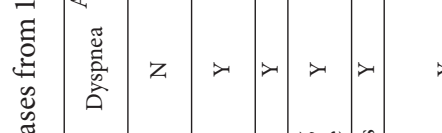

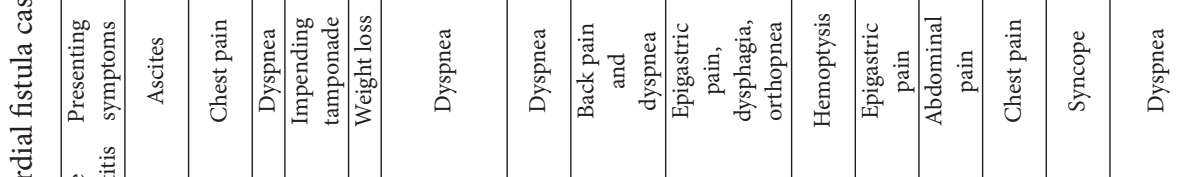

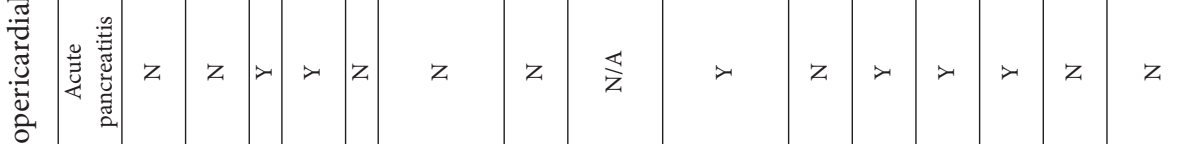

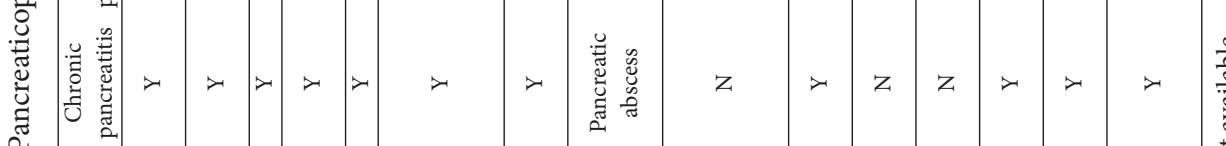

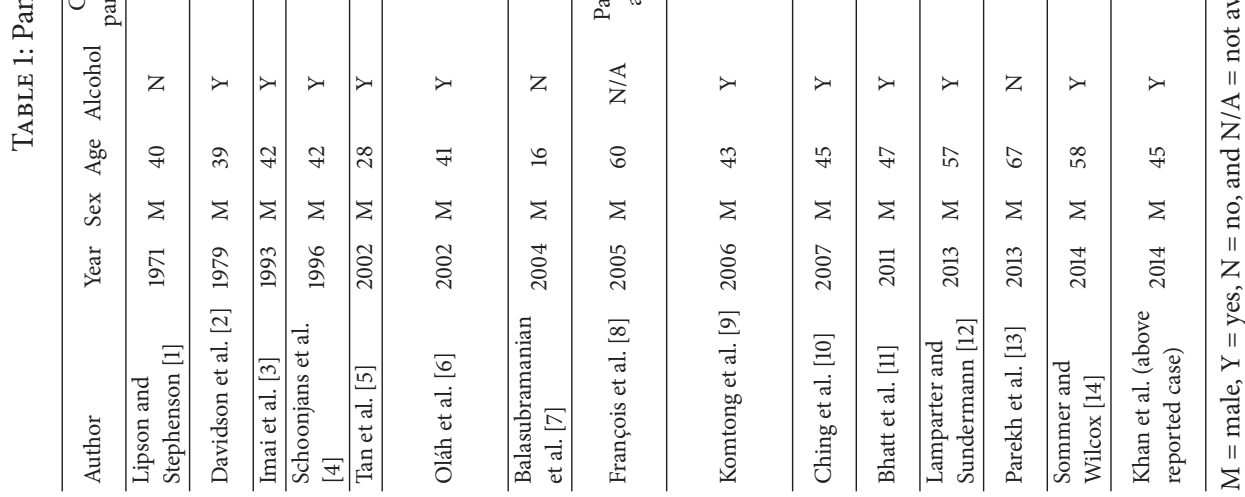


95\% confidence interval: 0.32 to 1.67 , and $p$ value $=0.46$ ) Of the patients presenting with cardiac tamponade, $50 \%$ had acute pancreatitis on admission $(\mathrm{RR}=1.25,95 \% \mathrm{CI}: 0.34$ to 4.48 , and $p$ value $=0.73)$. Pericardiocentesis was done in a total of 11 patients $(73.3 \%)$. Of the patients who underwent pericardiocentesis, eight patients recovered (72.7\% recovery). Endoscopic retrograde cholangiopancreatography (ERCP) was used in $40 \%$ of the patients to diagnose PPF. Computed tomography (CT) abdomen was used to make the diagnosis in $26.6 \%$ of patients. Two patients had CT angiogram of the chest to rule out pulmonary embolism that revealed suspected pancreaticopericardial fistula, which was later confirmed by MRCP. Three cases were diagnosed by direct surgical exploration of the pancreatic duct. For definitive treatment, six patients underwent surgery (40\%) with complete recovery in all six patients. Endoscopic retrograde cholangiopancreatography (ERCP) was done for four patients (26.6\% cases) of which two patients recovered completely and two patients died. Two patients underwent pericardial window only $(13.3 \%)$ of which only one patient recovered. One patient was managed conservatively without any invasive intervention. An external transhepatic drain was placed in one patient. Endoscopic ultrasound guided cystgastrostomy was done in one patient. All three patients had a complete recovery.

\section{Discussion}

A pancreatic fistula (PF) is defined as an abnormal connection between the pancreas and adjacent or distant organs, structures, and spaces [15]. PFs are classified as "internal" if the pancreatic duct communicates with the peritoneal or pleural cavity or any other hollow viscus and "external" if the pancreatic duct communicates with the skin [1]. The pathogenic mechanism of both internal and external PFs consists of disruption of the pancreatic duct secondary to pancreatitis or trauma $[16,17]$. Persistent leakage of pancreatic secretions can result in development of internal pancreatic fistulas to neighboring hollow viscus (colon, duodenum, stomach, and esophagus), body cavities (peritoneal, pleural, and mediastinal), and vasculature (splenic vein, portal vein, and aorta). If the communication occurs anteriorly into the peritoneal cavity, it results in pancreatic ascites. A posterior communication may track into the pleural cavity or mediastinum [18]. A mediastinal pseudocyst occurs if the secretions are confined to mediastinum [18]. However, penetration of the secretion through pleura into pleural cavities, pericardium, or bronchial tree results in pancreaticopleural, pancreaticopericardial, or pancreaticobronchial fistula, respectively.

5.1. Incidence. The incidence of $\mathrm{PFs}$ after pancreatic resection ranges from 10 to $29 \%$ depending upon the type of surgery and underlying pancreatic pathology $[15,16]$. The exact incidence of thoracopancreatic fistula and especially pancreaticopericardial fistula (PPF) is unknown. A review of English medical literature extending from 1965 to 1990 showed only 89 cases of thoracopancreatic fistulas [19]. The incidence of pancreaticopleural fistula has been reported in roughly $0.4 \%$ of patients with pancreatitis and $2.3-4.5 \%$ of patients presenting with pancreatic pseudocyst [20]. Our report indicates an increased incidence of pancreaticopericardial fistula in patients with existing history of chronic pancreatitis (73.3\%). Acute pancreatitis was present in $42 \%$ of patients with PPF on admission. It is interesting to note that, of the patients with acute pancreatitis, $66 \%$ did not have any history of chronic pancreatitis. This association could be due to an undetected chronic subclinical pancreatitis in this patient population. However, we cannot exclude the possibility of acute pancreatitis resulting in fistula to pericardium based on the available information.

5.1.1. Clinical Presentation. The clinical symptoms of thoracopancreatic fistula include cough, shortness of breath, chest pain, wheezing, palpitations, and dysphagia [21]. Patients with pancreaticopleural fistula can have unilateral and bilateral pleural effusions with dullness to percussion over the thorax and diminished breath sounds on physical examination. Our study indicates that patients with PPF are predominately male with median age of 43 years and a strong history of alcoholism (78\%). Dyspnea is the most common clinical symptom $(78 \%)$ followed by hypotension $(53 \%)$, chest pain $(50 \%)$, fever $(43 \%)$, abdominal pain $(26.6 \%)$, syncope $(6.6 \%)$, weight loss $(6.6 \%)$, and ascites (6.6\%). Cardiac tamponade was present in $53 \%$ of patients, and of all the patients with cardiac tamponade $75 \%$ had history of chronic pancreatitis $(\mathrm{RR}=$ $0.74,95 \%$ CI: 0.32 to 1.67 , and $p$ value $=0.46)$. Because of small sample size and the fact that PPF is a rare disease, the relative risk and confidence interval cannot be interpreted inaccurately. However, given that majority of patients with PPF had past medical history of chronic pancreatitis, a PPF should be suspected in patients with chronic pancreatitis presenting with cardiac tamponade.

5.1.2. Diagnosis. The diagnosis of thoracopancreatic fistula should be suspected in patients with history of pancreatitis, pancreatic trauma, or surgery who present with a combination of abdominal and chest symptoms (dyspnea, orthopnea, chest pain, wheezing, etc.). The diagnosis is established by the finding of high levels of amylase in extravasated pancreatic fluid and evidence of duct disruption on imaging. For pancreaticopleural fistulas, the pleural effusions are exudative and amylase-rich with pleural fluid amylase greater than the upper limit of normal for serum amylase or a pleural fluid to serum amylase ratio greater than 1.0 [22]. To date there is no published data regarding the cardiac fluid analysis in patients with pancreaticopericardial fistula. Our study indicates that the cardiac fluid in such patients is exudative and amylaserich in nature with pericardial fluid amylase to serum amylase ratio greater than 1.0. Our case and three more cases found an increased level of pericardial fluid lipase levels in patients with PPF.

5.2. Imaging. A number of imaging studies have been proposed to diagnose internal pancreatic fistulas. A CT scan of abdomen may demonstrate free fluid and walled-off collections in abdominal and thoracic cavities and changes of acute and chronic pancreatitis. However, a CT scan is 
diagnostic of pancreatic fistula only if performed immediately after an ERCP when it may demonstrate a fistulous tract [23]. MRCP is also another noninvasive method of diagnosing fistulas and should be performed if there is concern for adjacent complications (example is a pseudoaneurysm on CT) or if an ERCP is not performed. MRCP has advantage of guiding the clinical management by delineating the pancreatic duct injuries including those upstream of a complete duct disruption that would not be visualized on ERCP [24, 25]. ERCP provides direct evidence of a pancreatic fistula and is the test of choice if therapeutic pancreatic stenting is planned [26]. Unlike abdominal CT or MRCP, ERCP has the ability to demonstrate contrast filling the pancreatic ducts and extravasation in real time. ERCP has higher sensitivity and specificity for pancreatic duct leak as compared to CT scan [27]. Our report indicates that $40 \%$ of patients with pancreaticopericardial fistula were diagnosed by ERCP. CT abdomen was used to make the diagnosis in $26.6 \%$ of patients. Two patients had CT angiogram of the chest to rule out pulmonary embolism which revealed suspected pancreaticopericardial fistula which was later confirmed by MRCP. Three cases were diagnosed by direct surgical exploration of the pancreatic duct. In the above-mentioned patient the CT scan of chest on admission was done without contrast and failed to demonstrate the fistula. However CT angiogram of chest done in later hospital course demonstrated the fistula, indicating that contrast enhancement is required for visualization of fistulous tract.

5.3. Management. The management of pancreatic fistula is based on case reports and small observational studies [28]. The initial care depends on the presence of symptoms of abdominal pain, fever, and hypotension; presence of pancreatic necrosis on imaging and associated complications of infection; septic shock; and so forth. Reducing pancreatic stimulation by maintaining nil-by-mouth (NPO) status is part of initial management. Nasojejunal feeding to correct malnutrition is essential, and enteral nutrition is associated with lower incidence of infection, higher 30-day fistula closure rates, and shorter time to closure of postoperative pancreatic fistula as compared to total parenteral nutrition [2931]. Octreotide $100 \mathrm{mcg}$ three times daily in patients with high output fistula has been reported to be effective in reducing the fistula output, but it does not affect rate of fistula closure [29]. Fifty to sixty-five percent of internal fistulas close with supportive care [32]. In patients with persistent symptomatic fistula, additional interventions are required. Endoscopic treatment is preferred approach for management of most pancreatic fistulas. The goal of endoscopic therapy is internal drainage of pancreatic secretions, which thereby reduces flow through the fistula tract via placement of a pancreatic stent and/or pancreatic sphincterotomy $[33,34]$. In a case series, endoscopic therapy for pancreatic fistulas has been associated with success rates of $85-100 \%$ [32]. Surgery for pancreatic fistula is indicated when endoscopic management fails or is technically difficult and is associated with a success rate of $90 \%$ with an associated mortality of 6-9\% [32]. In case of pericardial tamponade, pericardiocentesis and pericardial drain placement becomes essential for initial stabilization of the patient followed by definitive treatment. All patients in our study who presented with acute tamponade had pericardial effusion drained. Four patients underwent ERCP guided stenting $(26.6 \%)$ of which two patients recovered while two patients died. Six patients underwent surgical treatment (40\%) with complete recovery in all six patients. Our patient underwent endoscopic ultrasound guided cystgastrostomy with complete recovery. Pericardial window was done as primary treatment in two patients $(13.3 \%)$ of which one patient survived. Two patients were managed conservatively and one patient eventually had a transhepatic drain placed. Both these patients recovered without any complications.

\section{Conclusion}

PPF is a rare complication of pancreatitis. To our knowledge this is the first paper on this subject discussing the patient demographics, clinical picture, diagnostic modalities, and treatment options. The data is limited owing to rarity of the disease and no randomized trials are available that guide the diagnosis and treatment options. Identification and reporting of cases are required to have a better understanding of the disease process, which can affect treatment modes and patient outcomes.

\section{Competing Interests}

The authors declare that they have no competing interests.

\section{References}

[1] J. D. Lipson and H. E. Stephenson Jr., "Pancreatitis complicated by pericardial effusion and cardiac tamponade. Recovery after prolonged cerebral anoxia," Archives of Surgery, vol. 103, no. 3, pp. 414-416, 1971.

[2] E. D. Davidson, J. T. Horney, and P. P. Salter III, "Internal pancreatic fistula to the pericardium and pleura," Surgery, vol. 85, no. 4, pp. 478-480, 1979.

[3] Y. Imai, M. Taniguchi, K. Tagawa et al., "A case of chronic pancreatitis complicated by massive pericardial and right pleural effusion," Gastroenterologia Japonica, vol. 28, no. 5, pp. 734-738, 1993.

[4] R. Schoonjans, K. Vandewoude, E. Cesmeli et al., "Recurrent pericardial effusion as a result of chronic pancreatitis. Successful treatment with somatostatin," Journal of Clinical Gastroenterology, vol. 23, no. 3, pp. 224-227, 1996.

[5] M. H. Tan, G. Kirk, P. Archibold, P. Kennedy, and M. C. Regan, "Cardiac compromise due to a pancreatic mediastinal pseudocyst," European Journal of Gastroenterology and Hepatology, vol. 14, no. 11, pp. 1279-1282, 2002.

[6] A. Oláh, A. S. Nagy, I. Rácz, and M. E. Gamal, "Cardiac tamponade as complication of pseudocyst in chronic pancreatitis," Hepato-Gastroenterology, vol. 49, no. 44, pp. 564-566, 2002.

[7] P. Balasubramanian, R. Jeyamani, S. Govil et al., "Pancreaticopericardial fistula: a rare complication of chronic pancreatitis," Indian Journal of Gastroenterology, vol. 23, no. 1, pp. 31-32, 2004.

[8] C. J. François, T. C. Demos, and N. Iqbal, "Pancreaticothoracic fistulas: imaging findings in five patients," Abdominal Imaging, vol. 30, no. 6, pp. 761-767, 2005. 
[9] S. Komtong, R. Chanatrirattanapan, P. Kongkam, R. Rerknimitr, and P. Kullavanijaya, "Mediastinal pseudocyst with pericardial effusion and dysphagia treated by endoscopic drainage," Journal of the Pancreas, vol. 7, no. 4, pp. 405-410, 2006.

[10] S.-S. Ching, M. M. Rao, A. Ali, S. Yeluri, A. G. Chalmers, and M. J. McMahon, "Chronic pancreatitis complicated by pancreaticopericardial fistula," Surgical Practice, vol. 11, no. 3, pp. 130-133, 2007.

[11] V. R. Bhatt, A. Koirala, R. V. Wetz, S. Kedia, P. Ghimire, and R. Bartaula, "Cardiac tamponade in acute pancreatitis," BMJ Case Reports, vol. 2011, 2011.

[12] S. Lamparter and H. Sundermann, "Swinging heart in acute pancreatitis," American Journal of the Medical Sciences, vol. 346, no. 2, pp. 160-161, 2013.

[13] P. Parekh, D. Howerton, and D. Johnson, "Not your everyday case of acute pancreatitis: a rare complication of a common diagnosis," ACG Case Reports Journal, vol. 1, no. 1, pp. 40-43, 2013.

[14] C. A. Sommer and C. M. Wilcox, "Pancreatico-pericardial fistula as a complication of chronic pancreatitis," F1000Research, vol. 3, article 31, 2014.

[15] C. Bassi, G. Butturini, E. Molinari et al., "Pancreatic fistula rate after pancreatic resection: the importance of definitions," Digestive Surgery, vol. 21, no. 1, pp. 54-59, 2004.

[16] G. G. Tsiotos, C. D. Smith, and M. G. Sarr, "Incidence and management of pancreatic and enteric fistulas after surgical management of severe necrotizing pancreatitis," Archives of Surgery, vol. 130, no. 1, pp. 48-52, 1995.

[17] J. L. Cameron, R. S. Kieffer, W. J. Anderson, and G. D. Zuidema, "Internal pancreatic fistulas: pancreatic ascites and pleural effusions," Annals of Surgery, vol. 184, no. 5, pp. 587-593, 1976.

[18] L. Kaman, A. Behera, R. Singh, and R. N. Katariya, "Internal pancreatic fistulas with pancreatic ascites and pancreatic pleural effusions: recognition and management," ANZ Journal of Surgery, vol. 71, no. 4, pp. 221-225, 2001.

[19] P. A. Lipsett and J. L. Cameron, "Internal pancreatic fistula," The American Journal of Surgery, vol. 163, no. 2, pp. 216-220, 1992.

[20] D. Semba, Y. Wada, Y. Ishihara, T. Kaji, A. Kuroda, and Y. Morioka, "Massive pancreatic pleural effusion: pathogenesis of pancreatic duct disruption," Gastroenterology, vol. 99, no. 2, pp. 528-532, 1990.

[21] D. C. Rockey and J. P. Cello, "Pancreaticopleural fistula: report of 7 patients and review of the literature," Medicine, vol. 69, no. 6, pp. 332-344, 1990.

[22] A. S. Fulcher, G. W. Capps, and M. A. Turner, "Thoracopancreatic fistula: clinical and imaging findings," Journal of Computer Assisted Tomography, vol. 23, no. 2, pp. 181-187, 1999.

[23] J. Joseph, S. Viney, P. Beck, C. Strange, S. A. Sahn, and G. S. Basran, "A prospective study of amylase-rich pleural effusions with special reference to amylase isoenzyme analysis," Chest, vol. 102, no. 5, pp. 1455-1459, 1992.

[24] J. S. Barkin, R. M. Ferstenberg, W. Panullo, H. D. Manten, and R. C. Davis Jr., "Endoscopic retrograde cholangiopancreatography in pancreatic trauma," Gastrointestinal Endoscopy, vol. 34, no. 2, pp. 102-105, 1988.

[25] A. S. Fulcher, M. A. Turner, J. A. Yelon et al., "Magnetic resonance cholangiopancreatography (MRCP) in the assessment of pancreatic duct trauma and its sequelae: preliminary findings," Journal of Trauma-Injury, Infection and Critical Care, vol. 48, no. 6, pp. 1001-1007, 2000.
[26] J. A. Soto, O. Alvarez, F. Múnera, N. L. Yepes, M. E. Sepúlveda, and J. M. Pérez, "Traumatic disruption of the pancreatic duct: diagnosis with MR pancreatography," American Journal of Roentgenology, vol. 176, no. 1, pp. 175-178, 2001.

[27] J. Devière, H. Bueso, M. Baize et al., "Complete disruption of the main pancreatic duct: endoscopic management," Gastrointestinal Endoscopy, vol. 42, no. 5, pp. 445-451, 1995.

[28] J. A. Blatnik and J. M. Hardacre, "Management of Pancreatic fistulas," Surgical Clinics of North America, vol. 93, no. 3, pp. 611617, 2013.

[29] S. L. Gans, H. L. van Westreenen, J. J. S. Kiewiet, E. A. J. Rauws, D. J. Gouma, and M. A. Boermeester, "Systematic review and meta-analysis of somatostatin analogues for the treatment of pancreatic fistula," British Journal of Surgery, vol. 99, no. 6, pp. 754-760, 2012.

[30] S. Klek, M. Sierzega, L. Turczynowski, P. Szybinski, K. Szczepanek, and J. Kulig, "Enteral and parenteral nutrition in the conservative treatment of pancreatic fistula: a randomized clinical trial," Gastroenterology, vol. 141, no. 1, pp. 157-163.el, 2011.

[31] D. K. Heyland, R. Dhaliwal, J. W. Drover, L. Gramlich, and P. Dodek, "Canadian clinical practice guidelines for nutrition support in mechanically ventilated, critically ill adult patients," Journal of Parenteral and Enteral Nutrition, vol. 27, no. 5, pp. 355-373, 2003.

[32] N. Alexakis, R. Sutton, and J. P. Neoptolemos, "Surgical treatment of pancreatic fistula," Digestive Surgery, vol. 21, no. 4, pp. 262-274, 2004.

[33] J. J. Telford, J. J. Farrell, J. R. Saltzman et al., "Pancreatic stent placement for duct disruption," Gastrointestinal Endoscopy, vol. 56, no. 1, pp. 18-24, 2002.

[34] R. A. Kozarek, T. J. Ball, D. J. Patterson, P. C. Freeny, J. A. Ryan, and L. William Traverso, "Endoscopic transpapillary therapy for disrupted pancreatic duct and peripancreatic fluid collections," Gastroenterology, vol. 100, no. 5, part 1, pp. 1362-1370, 1991. 


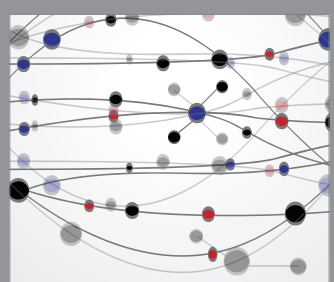

The Scientific World Journal
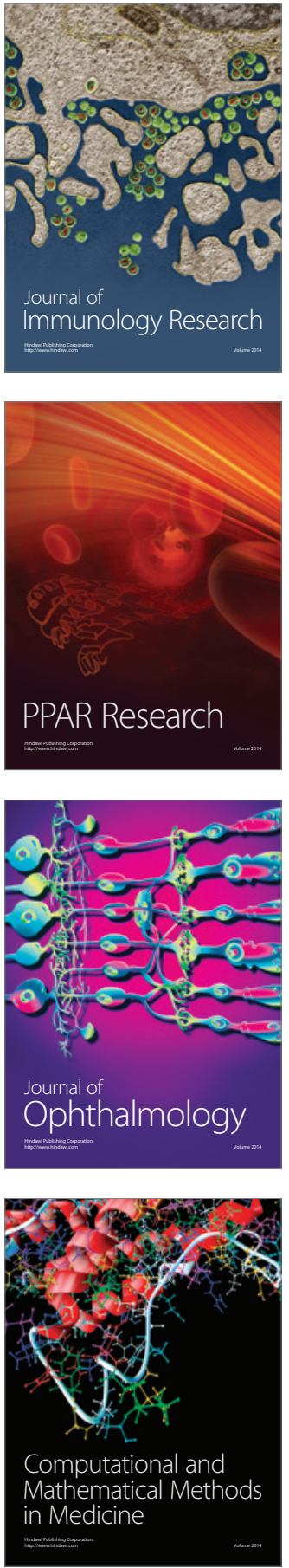

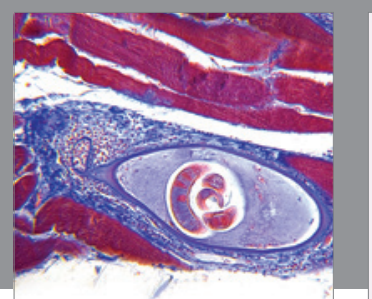

Gastroenterology Research and Practice

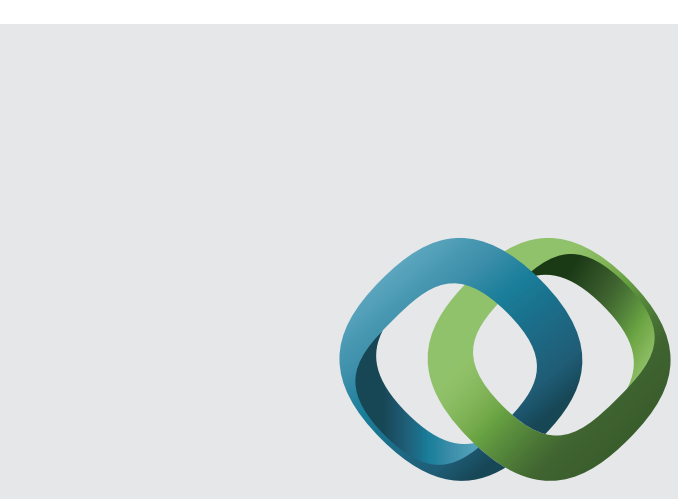

\section{Hindawi}

Submit your manuscripts at

http://www.hindawi.com
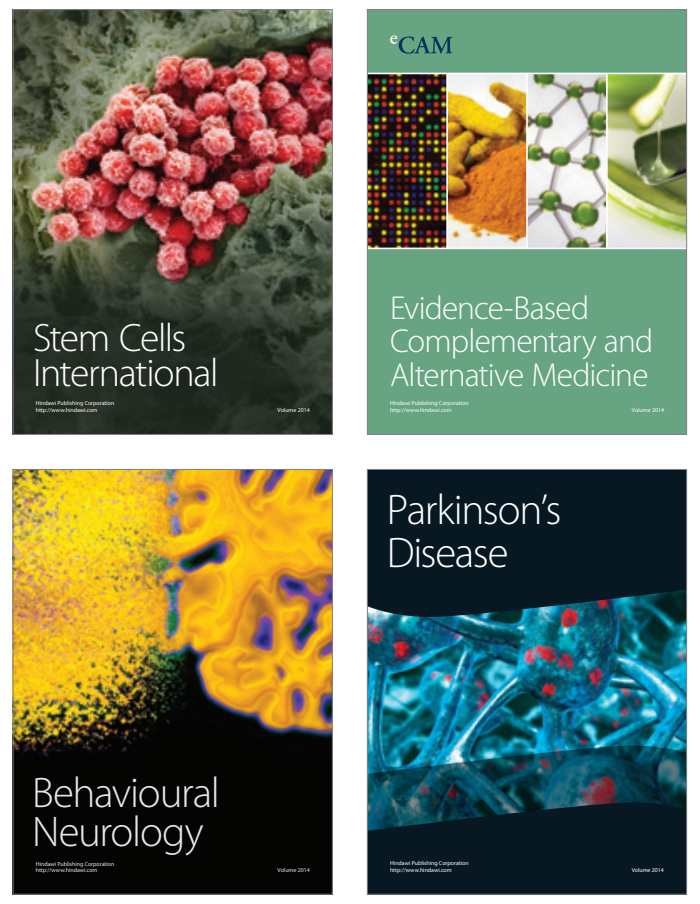
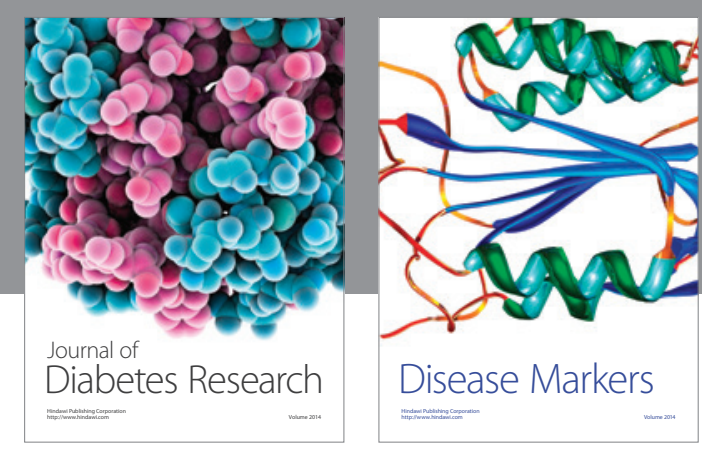

Disease Markers
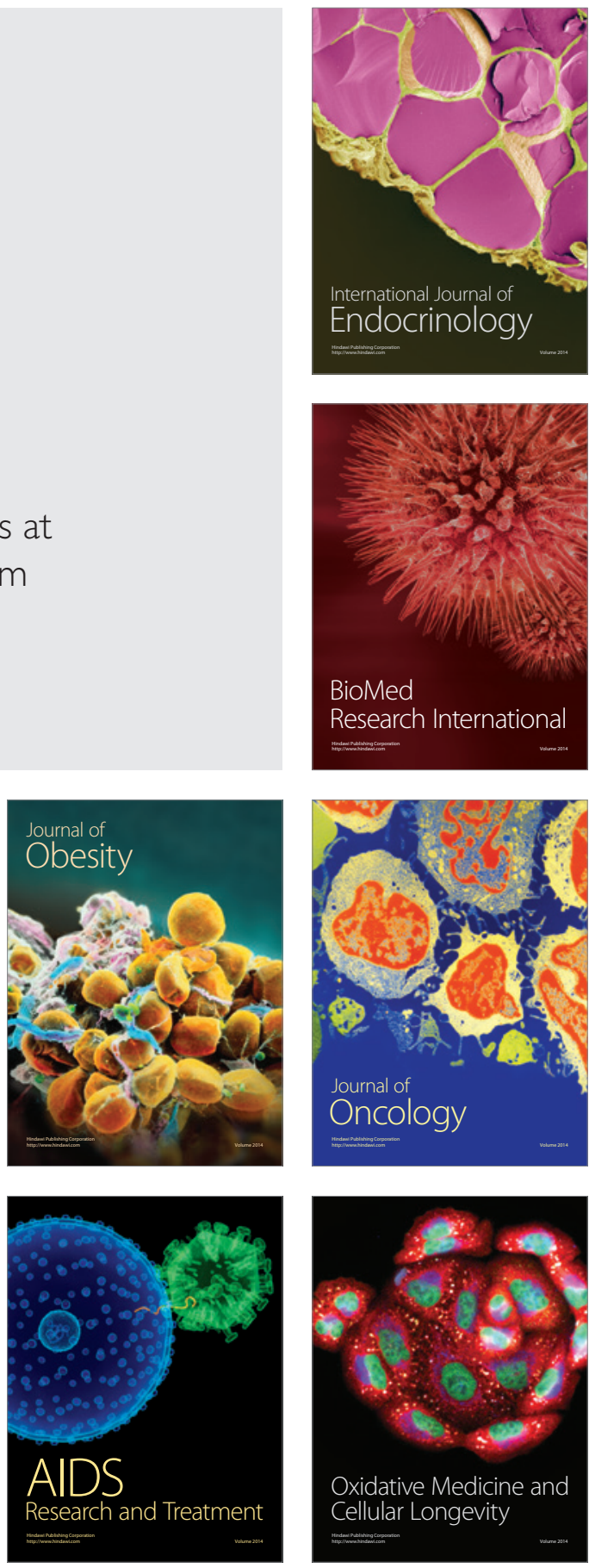